\title{
Difference in Activities of Phospholipase C for Hydrolysis of Lipid/alkanethiol Bilayers and Lipid Monolayers Formed on Au(111)
}

\author{
Daisuke HOBARA, Yasufumi YAMAMURA, ${ }^{\text {a }}$ Takayuki SASAKI, ${ }^{\mathrm{b}}$ and Takashi KAKIUCHI*
}

\begin{abstract}
Department of Energy and Hydrocarbon Chemistry, Graduate School of Engineering, Kyoto University (Kyoto 6068501, Japan)

aPresent address: Pioneering Research Laboratories, Toray Industries, Inc.

${ }^{b}$ Department of Chemistry and Biotechnology, Yokohama National University (Yokohama 240-8501, Japan), Present address: MEITEC corporation
\end{abstract}

Received July 23, 2001 ; Accepted September 4, 2001

\begin{abstract}
Double layer capacitance $\left(C_{\mathrm{dl}}\right)$ measurements have been used for in situ monitoring of phospholipase C (PLC) activity at phospholipid monolayers formed on $\mathrm{Au}(111)$ electrodes. The enzymatic hydrolysis of the phospholipid monolayer formed on a 1-hexanethiol self-assembled monolayer can be monitored as increase of $C_{\mathrm{dl}}$ of the electrode/solution interface. On the other hand, $C_{\mathrm{dl}}$ of Au(111) modified with 12-bis(11-mercaptoundecanoyl)-sn-glycero-3-phosphocholine monolayer is constant with time in the presence of PLC, indicating that PLC is not active for the monolayer. The difference in the PLC activity between the two systems suggests that flexibility of the lipid molecules at the surface is important for the enzymatic hydrolysis.
\end{abstract}

Key Words : Phospholipase C, Self-assembled Monolayer, Double Layer Capacitance, Au(111)

\section{Introduction}

The enzymatic hydrolysis of lipid bilayers by phospholipases at the air/water or the oil/water interfaces was markedly affected by the packing, the surface pressure, and the composition of the lipid layer. ${ }^{1,2)}$ We have previously reported that double layer capacitance $\left(C_{\mathrm{dl}}\right)$ is useful for measuring the activity of phospholipases $\mathrm{D}$ and $\mathrm{A}_{2}$ on phosphatidylcholine monolayers at the nitrobenzene/ water interface under the potential control. ${ }^{3-5)}$

Compared with the lipid monolayers at air/water or the oil/water interfaces, lipid monolayers and multilayers formed on metal surfaces have advantages of simpler preparation and high stability. In addition, a number of surface analytical techniques such as FTIR, ${ }^{6)}$ X-ray photoelectron spectroscopy (XPS), ${ }^{7)}$ atomic force microscopy, ${ }^{7-9)}$ SIMS, ${ }^{7)}$ and electrochemical methods ${ }^{10)}$ can be employed for studying interfacial activity of phospholipases on metal-supported lipid layers. The enzymatic reactions on lipid-modified metal surfaces may open a new route for the chemical modification of the surfaces utilizing the high specificity of enzymes.

In the present study, the phospholipase C (PLC) activity has been studied using two different lipid systems formed on $\mathrm{Au}(111)$. One is an $\mathrm{L}-\alpha$-dimyristoylphosphatidylcholine (DMPC) monolayer formed on a selfassembled monolayer (SAM) ${ }^{11)}$ of 1-hexanethiol (HTphospholipid bilayer). The other is a SAM of 12-bis ( 11 -mercaptoundecanoyl ) -sn-glycero- 3 -phosphocholine (MUDPC), which has two thiol groups at the end of the acyl groups and allows strong binding with the gold surface. The activity of PLC was studied by XPS and surface plasmon resonance (SPR) as well as $C_{\mathrm{dl}}$ measurements. Mirsky et $a l$. reported the study of the phospholipase $\mathrm{A}_{2}$
(PLA2) activity on phospholipid layers deposited on octadecanethiol-monolayer-formed gold electrodes using similar techniques. ${ }^{12.13)}$ In the hydrolysis of phospholipid by $\mathrm{PLA}_{2}$, it is known that the phospholipid molecule

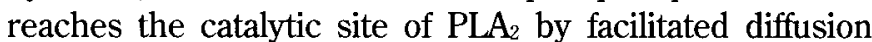
from the interfacial binding surface to the catalytic site of PLA through the hydrophobic channel of PLA ${ }_{2}{ }^{14)}$ It is not known if a similar mobility of a lipid molecule is required in the hydrolysis by PLC. The two systems used in the present study are expected to provide the information about the mechanism of the PLC hydrolysis regarding the accessibility of lipid molecules at the active site.

\section{Experimental}

PLC (Type IV from Bacillus cereus, Sigma), 1hexanethiol (HT, Aldrich), and DMPC (Sigma) were used without further purification. 12-bis (11-mercaptoundecanoyl)-sn-glycero-3-phosphocholine (MUDPC) was synthesized according to the procedures described previously ${ }^{15)}$ All other chemicals used were of reagent grade. Water was distilled and purified with a Milli-Q system (Millipore). Au(111) substrates were prepared by vacuum deposition of gold on mica ${ }^{16)}$ for $C_{\mathrm{dl}}$ measurements, XPS, and cyclic voltammetry. For SPR measurements, $50 \mathrm{~nm}$ layer of gold was vacuum-deposited on glass (SF 10 , Schott). ${ }^{17)}$

A $0.2 \mathrm{mM}$ DMPC vesicle solution was prepared by diluting a DMPC propanol solution with $20 \mathrm{mM}$ Tris- $\mathrm{HCl}$ buffer $(\mathrm{pH}=7.3)$. An HT SAM was formed by immersing the gold substrate into a $1 \mathrm{mM}$ solution of HT in ethanol for at least $24 \mathrm{~h}$. The gold substrate was then immersed into the DMPC vesicle solution for at least $6 \mathrm{~h}$ to form an HT-DMPC bilayer. The formation of the bilayer was 
confirmed by the $C_{\mathrm{d} 1}$ measurement. ${ }^{13,18)} C_{\mathrm{d} 1}$ decreased with time after addition of DMPC and reached a steady value at $80 \mathrm{~min}$. An MUDPC monolayer was formed by immersing the gold substrate into a $1 \mathrm{mM}$ solution of MUDPC in ethanol for at least $24 \mathrm{~h}$.

$C_{\mathrm{dl}}$ was measured in a $20 \mathrm{mM}$ Tris- $\mathrm{HCl}$ buffer solution (pH 7.3) containing $150 \mathrm{mM} \mathrm{NaCl}$ at room temperature by a frequency analyzer (Model 5010A, NF Electronic Instruments). An a.c. voltage of $40 \mathrm{~Hz}$ and $20 \mathrm{mV}$ (rms) was superimposed on the d.c. voltage of $0.0 \mathrm{~V}$ (vs. $\mathrm{Ag}$ / $\mathrm{AgCl} \mathrm{KCl}$ sat.). PLC was added to a concentration of 10 unit $\mathrm{ml}^{-1}$ to study the PLC activity. XPS measurements were made using a model-500 MT (ULVAC-PHI) with $\mathrm{Mg}$-K $\alpha$ radiation $(15 \mathrm{kV}, 400 \mathrm{~W})$ at less than $7 \times 10^{-7} \mathrm{~Pa}$. A home-built instrument employing a $632.8 \mathrm{~nm}$ He-Ne laser $^{19)}$ was used for SPR measurements. Time-dependence of the reflectivity was measured at a fixed angle that is $1.5^{\circ}$ lower than the plasmon minimum. The plasmon minimum typically appeared at $\sim 56^{\circ}$. All SPR measurements were carried out at room temperature.

\section{Results and Discussion}

Figure 1a shows time-dependence of $C_{\mathrm{dl}}$ of $\mathrm{Au}(111)$ modified with the HT-DMPC bilayer after addition of the PLC vesicle solution. Before addition of PLC, $C_{\mathrm{dl}}$ was constant with time. After the addition of PLC in the aqueous phase, $C_{\text {tl }}$ gradually increased and reached a constant value at about $100 \mathrm{~min}$. This increase in $C_{\mathrm{dl}}$ was attributable to the decrease in the bilayer thickness caused by the hydrolysis of DMPC by PLC; PLC selectively hydrolyzed the glycerol-phosphate ester bond of DMPC to produce phosphocholine, that diffused away from the surface. The adsorption of positively charged PLC molecules (at pH 7) may also contribute to the increase of $C_{\mathrm{dl}}$ in Fig. 1a. The initial rapid increase of $C_{\mathrm{dl}}(t<10 \mathrm{~min})$ might reflect the PLC adsorption.

Figure $1 \mathrm{~b}$ shows a time-dependence of the reflectivity of a gold film modified with the HT-DMPC bilayer measured by SPR. The reflectivity rapidly increased with time after the addition of PLC $(t<30 \mathrm{~min})$, indicating the increase of the thickness of the membrane. The increase of the reflectivity indicates the adsorption of PLC or proteins contained as impurities. The reflectivity, then, decreased $(30 \mathrm{~min}<t<40 \mathrm{~min}$ ) and reached a constant value ( $t>40 \mathrm{~min}$ ), suggesting the decrease in the bilayer thickness by the enzymatic hydrolysis. It is interesting that the time-scale between the $C_{\mathrm{dr}}$ measurement and SPR (Figs. 1a and $1 b$ ) is different. The gold film evaporated on glass is rougher than that on mica. The shorter
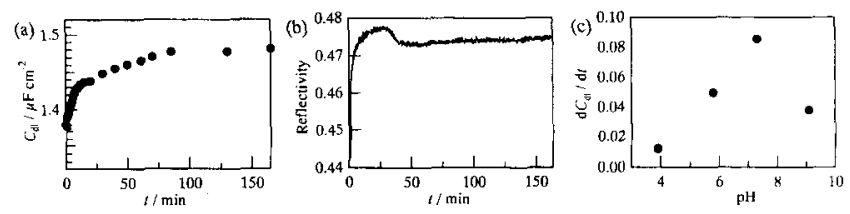

Fig. 1 Time dependence of (a) $C_{\text {dl }}$ of the HT-DMPC bilayer, and (b) the reflectivity of a gold film modified with the HTDMPC bilayer measured by SPR. PLC was added at $t=0$. (c) $\mathrm{pH}$ dependence of the rate of change in $C_{d 1}$ at $10 \mathrm{~min}$ after the PLC injection. time required for reaching a constant value in the SPR measurement on the glass substrates is probably due to the increased number of the defects that can be the starting points for the enzymatic hydrolysis. ${ }^{20)}$

Time-dependence of $C_{\mathrm{dl}}$ of the HT-DMPC bilayer after the injection of PLC was measured in solutions having different $\mathrm{pH}$ values. The slope of the $C_{\mathrm{dl}}$ curve at $t=10$ min was plotted against $\mathrm{pH}$ of the solution in Fig. 1c. The plot shows a maximum at $\mathrm{pH}$ 7. The $\mathrm{pH}$ dependence is similar to that of the PLC activity toward micelles of phospholipid. ${ }^{21)}$ This clearly shows that the change in $C_{\mathrm{dl}}$ is caused by the enzymatic hydrolysis of the bilayer.

Figure 2 shows XPS spectra of the Au(111) surfaces where the HT-DMPC bilayers are formed. After the formation of the HT-DMPC bilayer, a peak appeared at 400 $\mathrm{eV}$ in the XPS spectrum of the $\mathrm{N}(1 \mathrm{~s})$ region (Curve 2 in Fig. 2a), whereas no peak was observed with the HT SAM in the same region (Curve 1 in Fig. 2a), indicating that the peak originates from the nitrogen of DMPC. The peak at $400 \mathrm{eV}$ decreased after the PLC treatment at $\mathrm{pH} 7$ for $24 \mathrm{~h}$. XPS spectra of the $\mathrm{O}(1 \mathrm{~s})$ region showed similar results (Fig. 2b) except that the peak which is probably from impurities appeared at $536 \mathrm{eV}$ in the XPS spectrum of the HT SAM (Curve 1 Fig. 2b). The decrease in the XPS signals after the PLC treatment is attributable to the loss of phosphocholine by the PLC hydrolysis of the DMPC layer. The persistence of the peak from $N(1 \mathrm{~s})$ after the PLC treatment indicates that the hydrolysis did not proceed completely.

Figure 3 shows a cyclic voltammogram of the reduc-
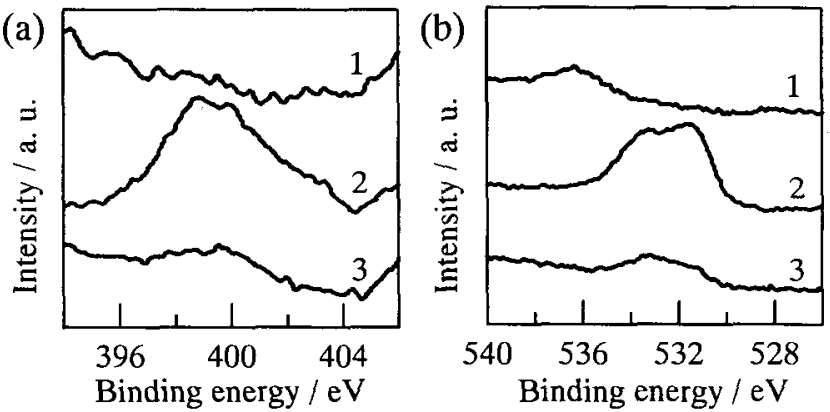

Fig. 2 XPS spectra of (a) N $1 \mathrm{~s}$, and (b) O $1 \mathrm{~s}$ regions for HT monolayers (curve 1), HT-DMPC bilayers (curve 2), and the bilayer after immersion in a PLC solution for $24 \mathrm{~h}$ (curve 3).

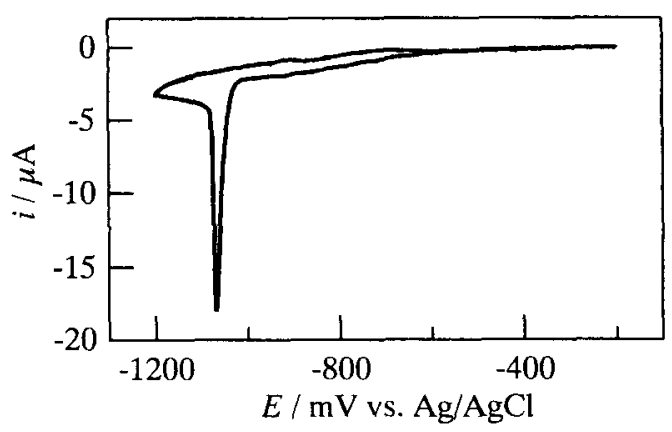

Fig. 3 Cyclic voltammogram for the reductive desorption of an MUDPC monolayer on Au(111). Scan rate: $20 \mathrm{mV} \mathrm{s}^{-1}$. 


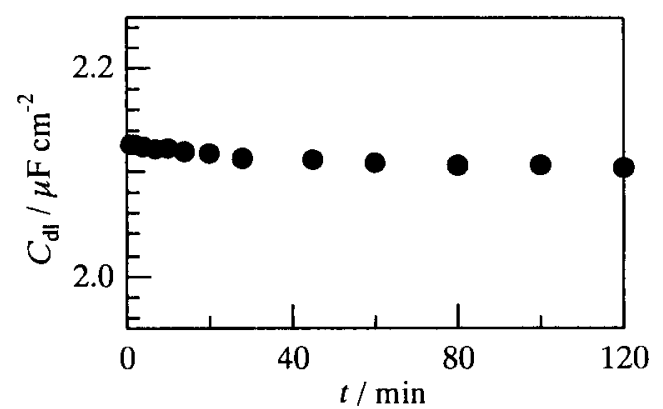

Fig. 4 Time dependence of $C_{\mathrm{dl}}$ of an MUDPC monolayer after the addition of PLC.

tive desorption of the MUDPC monolayer formed on Au (111). A sharp reduction peak of the adsorbed MUDPC appeared at $-1060 \mathrm{mV}$ in the cathodic scan. The charge passed under the peak was $105 \mu \mathrm{Ccm}^{-2}$ which is comparable to that for the reduction peak of alkanethiol SAMs on $\mathrm{Au}(111){ }^{22,23)}$ suggesting that the alkyl chains of MUDPC are closely packed on Au(111). Taking the bulkiness of the molecule into account, it is most likely that both of the two thiol groups in a MUDPC molecule are bound to the $\mathrm{Au}(111)$ surface. In such a case, the packing density of MUDPC can be calculated to be 0.44 $\mathrm{nm}^{2}$ per molecule by assuming that the sulfur atoms adopt a $(\sqrt{3} \times \sqrt{3}) \mathrm{R} 30^{\circ}$ structure on $\mathrm{Au}(111)$. The narrow peak $(\mathrm{fwhm}=17 \mathrm{mV})$ suggests the presence of a strong attractive interaction between the adsorbed molecules $^{24)}$ and close packing of MUDPC on Au(111).

Figure 4 shows a time-dependence of $C_{\mathrm{dl}}$ of $\mathrm{Au}(111)$ modified with an MUDPC monolayer after the addition of PLC in the solution. In contrast to the case of the HTDMPC bilayer, $C_{\mathrm{dl}}$ is almost constant with time. XPS spectra of the $P(2 p)$ region showed no marked change in the intensity after the PLC treatment (data not shown). The MUDPC monolayer is not, therefore, likely to be hydrolyzed by PLC.

The packing density of the MUDPC monolayer is similar to that of the lipid monolayer of the alkanethiolDMPC bilayer. ${ }^{25}$ ) The difference between the MUDPC monolayer and the HT-DMPC bilayer is the mobility of the lipid. The strong interaction would prevent the MUDPC molecules from accessing to the PLC active site, which is partially buried inside the enzyme. ${ }^{26)}$ Interestingly, Gaber et al. reported that PLC can hydrolyze the DMPC molecules covalently attached to a silicon wafer with the packing density being $1.2 \mathrm{~nm}^{2}$ per molecule. ${ }^{7)}$ In this case, the lower packing density seems to allow PLC to form an ES complex for the hydrolysis of lipid molecules strongly bound to the surface. Binary SAMs composed of MUDPC and alkanethiols such as 6mercapto- 1 -hexanol, 11 -mercapto- 1 -undecanol , 3 mercaptopropionic acid, 11-mercaptoundecanoic acid, and HT having several different surface compositions were prepared to study the PLC activity for lipid layers having lower packing densities. However, any results that indi- cate the enzymatic hydrolysis have not been obtained by $C_{\mathrm{dl}}$ measurements. Although more detail studies on the PLC activity for the binary systems are necessary, the limited flexibility of the MUDPC molecule due to the relatively short alkyl chain length probably prevents the enzymatic hydrolysis of the lipid.

\section{References}

1) R. Verger and G. H. d. Haas, Annu. Rev. Biophys. Bioeng. 5, 77 (1976).

2) E. A. Dennis, ed., Method in Enzymology, Vol. 197, Academic Press Inc., San Diego (1991).

3) T. Kondo, T. Kakiuchi, and M. Senda, Biochim. Biophys. Acta, 1124, 1 (1992).

4) T. Kondo, T. Kakiuchi, and M. Senda, Bioelectrochem. Bioenerg., 34, 93 (1994).

5) T. Kondo and T. Kakiuchi, Bioelectrochem. Bioenerg., 36, 53 (1995).

6) M. Grandbois, B. Desbat, and C. Salesse, Biophys. Chem., 88, $127(2000)$.

7) D. C. Turner, B. M. Peek, T. E. Wertz, D. D. Archibald, R. E. Geer, and B. P. Gaber, Langmuir, 12, 4411 (1996).

8) M. Grandbois, H. Clausen-Schaumann, and H. Gaub, Biophys. J., 74, 2398 (1998).

9) F. Noll, C. May, and H. Kindl, Biophys. Chem., 86, 29 (2000).

10) S. Chen and H. D. Abruña, Langmuir, 13, 5969 (1997).

11) A. Ulman, Chem. Rev., 96, 1533 (1996).

12) V. M. Mirsky, C. Krause, and K. D. Heckmann, Thin Solid Films, 284-285, 939 (1996).

13) V. M. Mirsky, M. Mass, C. Krause, and O. S. Wolfbeis, Anal. Chem., 70, 3674 (1998).

14) D. L. Scott, S. P. White, Z. Otwinowski, W. Yuan, M. H. Gelb, and P. B. Sigler, Science, 250, 1541 (1990).

15) N. K. P. Samuel, M. Singh, K. Yamaguchi, and S. L. Regen, J. Am. Chem. Soc., 107, 42 (1985).

16) D. Hobara, M. Ota, S. Imabayashi, K. Niki, and T. Kakiuchi, J. Electroanal. Chem., 444, 113 (1998).

17) C. A. Gross, D. H. Charych, and M. Madjda, Anal. Chem., 63, 85 (1991).

18) A. L. Plant, M. Gueguetchkeri, and W. Yap, Biophys. J., 67, 1126 (1994).

19) A. Shirakami, M. Yamamoto, D. Hobara, and T. Kakiuchi, in preparation.

20) M. Grandbois, J. Dufourcq, and C. Salesse, Thin Solid Films, 284-285, 743 (1996).

21) B. R. Eaton and E. A. Dennis, Arch. Biochem. Biophys., 176, 604 (1976).

22) D.F. Yang, C. P. Wilde, and M. Morin, Langmuir, 12, 6570 (1996).

23) C.-J. Zhong and M. D. Porter, J. Electroanal. Chem., 425, 147 (1997).

24) T. Kakiuchi, H. Usui, D. Hobara, and M. Yamamoto, in preparation.

25) J. B. Hubbard, V. Silin, and A. L. Plant, Biophys. Chem., 75, 163 (1998).

26) E. Hough, L. K. Hansen, B. Birknes, K. Jynge, S. Hansen, A. Hordvik, C. Little, E. Dodson, and Z. Derewenda, Nature, 338, 357 (1989). 